\title{
Extremal Matching Energy and the Largest Matching Root of Complete Multipartite Graphs
}

\author{
Xiaolin Chen and Huishu Lian \\ Department of Mathematics, China University of Mining and Technology, Xuzhou 221116, China \\ Correspondence should be addressed to Huishu Lian; 1hs6803@126.com
}

Received 15 February 2019; Accepted 4 April 2019; Published 16 April 2019

Academic Editor: Dimitri Volchenkov

Copyright (c) 2019 Xiaolin Chen and Huishu Lian. This is an open access article distributed under the Creative Commons Attribution License, which permits unrestricted use, distribution, and reproduction in any medium, provided the original work is properly cited.

\begin{abstract}
The matching energy $M E(G)$ of a graph $G$ was introduced by Gutman and Wagner, which is defined as the sum of the absolute values of the roots of the matching polynomial $m(G, x)$. The largest matching root $\lambda_{1}(G)$ is the largest root of the matching polynomial $m(G, x)$. Let $K_{n_{1}, n_{2}, \ldots, n_{r}}$ denote the complete $r$-partite graph with order $n=n_{1}+n_{2}+\ldots+n_{r}$, where $r>1$. In this paper, we prove that, for the given values $n$ and $r$, both the matching energy $M E(G)$ and the largest matching root $\lambda_{1}(G)$ of complete $r$-partite graphs are minimal for complete split graph $C S(n, r-1)$ and are maximal for Turán graph $T(n, r)$.
\end{abstract}

\section{Introduction}

Let $G$ be a finite simple graph of order $n$ with vertex set $V(G)$ and edge set $E(G)$. A matching of $G$ is a set of independent edges in $G$, and a $k$-matching of $G$ is a matching of $G$ that has exactly $k$ edges. By $m_{k}(G)$ we denote the number of $k$ matchings in $G$. It is easy to verify that, for $k<0$ and $k>$ $\lceil n / 2\rceil, m_{k}(G)=0$. And when $k=1, m_{1}(G)$ is the size of $G$. For convenience, we define $m_{0}(G)=1$. The matching polynomial $m(G, x)[1-3]$ of a graph $G$ is defined as

$$
m(G, x)=\sum_{k=0}^{\lfloor n / 2\rfloor}(-1)^{k} m_{k}(G) x^{n-2 k}
$$

The matching polynomial has been widely studied and has many important applications in statistical physics and chemistry; see [1-4]. The largest root of the matching polynomial $m(G, x)$, denoted by $\lambda_{1}(G)$, is called the largest matching root of $G$. The matching root has been much studied in algebraic combinatorics (see the book [5]). In [6], Fisher and Ryan deduced some bounds for $\lambda_{1}(G)$. Afterwards, many results about the largest matching root have been obtained; see $[7-12]$.

In [13], Gutman and Wagner introduced the matching energy (ME) of a graph $G$, which is defined as the sum of the absolute values of the roots of the matching polynomial of $G$. Note that the concept of the energy $E(G)$ of a simple undirected graph $G$ was introduced by Gutman in [14]. Afterwards, there have been lots of research papers on this topic. A systematic study of this topic can be found in the book [15]. In [13], Gutman and Wagner pointed out that the matching energy is a quantity of relevance for chemical applications. Moreover, they arrived at the simple relation

$$
\operatorname{TRE}(G)=E(G)-M E(G)
$$

where $\operatorname{TRE}(G)$ is the so-called "topological resonance energy" of the graph $G$. For more information about the applications of the matching energy, we refer the readers to $[16,17]$. Recently, there have been some results on the matching energy of graphs; see [18-31]. In spite of this, few results are known for the matching energy of complete multipartite graphs. Note that Stevanović et al. [32] studied the energy of complete multipartite graphs, which inspires us to study the matching energy of complete multipartite graphs.

For a fixed value $r>1$, let $K_{n_{1}, n_{2}, \ldots, n_{r}}$ denote the complete $r$-partite graph with order $n=n_{1}+n_{2}+\ldots+n_{r}$. There are two important complete $r$-partite graphs which have many implications in graph theory. One is complete split graph, denoted by $\operatorname{CS}(n, r-1)$, which is defined as $\operatorname{CS}(n, r-1) \cong$ $K_{n-r+1,1, \ldots, 1}$. The other is Turán graph $T(n, r)$, which is defined 
as the complete $r$-partite graph $K_{n_{1}, n_{2}, \ldots, n_{r}}$ satisfying $\left|n_{i}-n_{j}\right| \leq$ 1 for any two distinct numbers $1 \leq i, j \leq r$.

In this paper, we show that, among all the complete $r$ partite graphs with given order $n$, the graph with minimal matching energy is the complete split graph $C S(n, r-1)$ and the graph with maximal matching energy is Turán graph $T(n, r)$. Moreover, we prove that, among all the complete $r$ partite graphs with given order $n$, the graph with minimal $\lambda_{1}(G)$ is the complete split graph $C S(n, r-1)$ and the graph with maximal $\lambda_{1}(G)$ is Turán graph $T(n, r)$. In fact, we deduce the following theorems.

Theorem 1. For the fixed values $n$ and $r, \operatorname{ME}\left(K_{n_{1}, n_{2}, \ldots, n_{r}}\right) \geq$ $\operatorname{ME}(C S(n, r-1))$ and equality holds if and only if $K_{n_{1}, n_{2}, \ldots, n_{r}} \cong$ $C S(n, r-1)$.

Theorem 2. For the fixed values $n$ and $r, M E\left(K_{n_{1}, n_{2}, \ldots, n_{r}}\right) \leq$ $\operatorname{ME}(T(n, r))$ and equality holds if and only if $K_{n_{1}, n_{2}, \ldots, n_{r}} \cong$ $T(n, r)$.

Theorem 3. For the fixed values $n$ and $r, \lambda_{1}\left(K_{n_{1}, n_{2}, \ldots, n_{r}}\right) \geq$ $\lambda_{1}(C S(n, r-1))$ and equality holds if and only if $K_{n_{1}, n_{2}, \ldots, n_{r}} \cong$ $C S(n, r-1)$.

Theorem 4. For the fixed values $n$ and $r, \lambda_{1}\left(K_{n_{1}, n_{2}, \ldots, n_{r}}\right) \leq$ $\lambda_{1}(T(n, r))$ and equality holds if and only if $K_{n_{1}, n_{2}, \ldots, n_{r}} \cong T(n$, $r)$.

In Section 2, we prove Theorems 1 and 2. In Section 3, we give the proofs of Theorems 3 and 4 .

\section{Proofs of Theorems 1 and 2}

In this section, we first recall an integral formula for computing the value of matching energy which is also given by Gutman and Wagner [13].

Definition 5 (see [13]). Let $G$ be a simple graph. The matching energy of $G$ is

$$
M E(G)=\frac{2}{\pi} \int_{0}^{\infty} \frac{1}{x^{2}} \ln \left(\sum_{k \geq 0} m_{k}(G) x^{2 k}\right) d x
$$

By this definition, we can deduce that if $m_{k}(G) \geq m_{k}(H)$ for every $k \geq 1$, then $M E(G) \geq M E(H)$. Owing to the above observation, we derive the following lemma which is critical to the proofs of Theorems 1 and 2.

Lemma 6. For a complete r-partite graph $K_{n_{1}, \ldots, n_{i}, \ldots, n_{j}, \ldots, n_{r}}$ with $n_{j}-2 \geq n_{i}$, we have $m_{1}\left(K_{n_{1}, \ldots, n_{i}, \ldots, n_{j}, \ldots, n_{r}}\right)<m_{1}\left(K_{n_{1}, \ldots, n_{i}+1, \ldots, n_{j}-1, \ldots, n_{r}}\right)$, and $m_{k}\left(K_{n_{1}, \ldots, n_{i}, \ldots, n_{j}, \ldots, n_{r}}\right) \leq m_{k}\left(K_{n_{1}, \ldots, n_{i}+1, \ldots, n_{j}-1, \ldots, n_{r}}\right)$, for $2 \leq$ $k \leq n / 2$.

Proof. Let $G$ denote the graph $K_{n_{1}, n_{2}, \ldots, n_{r}}$. Let $V_{1}, V_{2}, \ldots, V_{r}$ be the $r$ partition classes with $\left|V_{i}\right|=n_{i}$ for $1 \leq i \leq r$ and $V_{i}=$ $\left\{v_{1}^{i}, v_{2}^{i}, \ldots, v_{n_{i}}^{i}\right\}$. Without loss of generality, we just prove the case $n_{2}-2 \geq n_{1}$.

For $k=1, m_{1}\left(K_{n_{1}+1, n_{2}-1, \ldots, n_{r}}\right)-m_{1}\left(K_{n_{1}, n_{2}, \ldots, n_{r}}\right)=\left(n_{1}+\right.$ 1) $\left(n_{2}-1\right)-n_{1} n_{2}=n_{2}-n_{1}-1>0$. For $2 \leq k \leq$ $n / 2$, we will prove this lemma by induction on the number $n_{1}$. For the case $n_{1}=1$, we first delete the vertex $v_{1}^{2}$ and the edges incident to it from $K_{1, n_{2}, \ldots, n_{r}}$, and then we get a new graph $G^{\prime}$ which is isomorphic to $K_{1, n_{2}-1, \ldots, n_{r}}$. Next, we add a new vertex $u$ into the graph $G^{\prime}$ and add some edges such that we get a graph $G^{\prime \prime}$ which is isomorphic to $K_{2, n_{2}-1, \ldots, n_{r}}$. In other words, these operations can be thought as the vertex $v_{1}^{2}$ being moved from the second partition class to the first partition class. Thus, for convenience, we denote the new vertex $u$ by $v_{1}^{2}$. Now, we compare the number of $k$ matchings between $G$ and $G^{\prime \prime}$. Choosing a $k$-matching $M$ in $G$, if the edge $\left(v_{1}^{1}, v_{1}^{2}\right)$ lies in the matching $M, M$ is not a $k$-matching of $G^{\prime \prime}$. Otherwise, the matching $M$ is a $k$ matching of $G^{\prime \prime}$. For a $k$-matching $M^{\prime}$ in $G^{\prime \prime}$, when there exists an edge between $v_{1}^{2}$ and $\left\{v_{2}^{2}, \ldots, v_{n_{2}}^{2}\right\}, M^{\prime}$ is not in $G$. Otherwise, $M^{\prime}$ is a $k$-matching of $G$. It follows that $m_{k}\left(G^{\prime \prime}\right)-$ $m_{k}(G)=\left(n_{2}-1\right) m_{k-1}\left(K_{1, n_{2}-2, n_{3}, \ldots, n_{r}}\right)-m_{k-1}\left(K_{n_{2}-1, n_{3}, \ldots, n_{r}}\right)$. Since $m_{k-1}\left(K_{1, n_{2}-2, n_{3}, \ldots, n_{r}}\right) \geq m_{k-1}\left(K_{n_{2}-1, n_{3}, \ldots, n_{r}}\right)$ and $n_{2}-1 \geq 1$, we get $m_{k}(G) \leq m_{k}\left(G^{\prime}\right)$.

Given $t \geq 2$, suppose that the lemma is right for $n_{1}=$ $t-1$, that is, $m_{k}\left(K_{t-1, n_{2}, \ldots, n_{r}}\right) \leq m_{k}\left(K_{t, n_{2}-1, \ldots, n_{r}}\right)$ for $2 \leq k \leq$ $n / 2$. For $n_{1}=t$, just like the above operations, we move the vertex $v_{1}^{2}$ from $V_{2}$ into $V_{1}$, then we get a new graph $G^{\prime}$ which is isomorphic to $K_{t+1, n_{2}-1, \ldots, n_{r}}$. For a $k$-matching $M$ in $G$, when there exists an edge between $\left\{v_{1}^{1}, \ldots, v_{t}^{1}\right\}$ and $v_{1}^{2}$ in the matching $M, M$ is not in $G^{\prime}$. Otherwise, the matching $M$ is a $k$-matching of $G^{\prime}$. For a $k$-matching $M^{\prime}$ in $G^{\prime}$, when there exists an edge between $v_{1}^{2}$ and $\left\{v_{2}^{2}, \ldots, v_{n_{2}}^{2}\right\}, M^{\prime}$ is not in $G$. Otherwise, $M^{\prime}$ is a $k$-matching of $G$. Then $m_{k}\left(G^{\prime}\right)-$ $m_{k}(G)=\left(n_{2}-1\right) m_{k-1}\left(K_{t, n_{2}-2, \ldots, n_{r}}\right)-t m_{k-1}\left(K_{t-1, n_{2}-1, \ldots, n_{r}}\right)$. Since $n_{2}-1>t, m_{k-1}\left(K_{t-1, n_{2}-1, \ldots, n_{r}}\right) \leq m_{k-1}\left(K_{t, n_{2}-2, \ldots, n_{r}}\right)$, then $m_{k}\left(G^{\prime}\right)-m_{k}(G) \geq 0$. Thus, we complete the proof.

From this lemma, we give the proofs of Theorems 1 and 2.

Proof of Theorem 1. Suppose that $K_{n_{1}, n_{2}, \ldots, n_{r}}$ is the complete multipartite graph with the smallest matching energy. If there are two parameters $n_{i}$ and $n_{j}$ satisfying $n_{j} \geq n_{i}>1$, then $\left(n_{j}+1\right)-\left(n_{i}-1\right) \geq 2$. From Lemma 6, we get $m_{1}\left(K_{n_{1}, \ldots, n_{i}, \ldots, n_{j}, \ldots, n_{r}}\right)>m_{1}\left(K_{n_{1}, \ldots, n_{i}-1, \ldots, n_{j}+1, \ldots, n_{r}}\right)$, and $m_{k}\left(K_{n_{1}, \ldots, n_{i}, \ldots, n_{j}, \ldots, n_{r}}\right) \geq m_{k}\left(K_{n_{1}, \ldots, n_{i}-1, \ldots, n_{j}+1, \ldots, n_{r}}\right)$ for $2 \leq k \leq n / 2$. It implies that $\operatorname{ME}\left(K_{n_{1}, \ldots, n_{i}, \ldots, n_{j}, \ldots, n_{r}}\right)>$ $\operatorname{ME}\left(K_{n_{1}, \ldots, n_{i}-1, \ldots, n_{j}+1, \ldots, n_{r}}\right)$. It is a contradiction. Hence, $K_{n_{1}, n_{2}, \ldots, n_{r}}$ satisfies that all parameters equal 1 except one parameter equal to $n-r+1$. Thus, $K_{n_{1}, n_{2}, \ldots, n_{r}} \cong C S(n, r-1)$.

Proof of Theorem 2. Suppose that $K_{n_{1}, n_{2}, \ldots, n_{r}}$ is the complete multipartite graph with the largest matching energy. If there are two parameters $n_{i}$ and $n_{j}$ satisfying $n_{j} \geq n_{i}+2$, from Lemma 6 , we get $m_{1}\left(K_{n_{1}, \ldots, n_{i}, \ldots, n_{j}, \ldots, n_{r}}\right)<$ $m_{1}\left(K_{n_{1}, \ldots, n_{i}+1, \ldots, n_{j}-1, \ldots, n_{r}}\right)$, and $m_{k}\left(K_{n_{1}, \ldots, n_{i}, \ldots, n_{j}, \ldots, n_{r}}\right) \leq$ $m_{k}\left(K_{n_{1}, \ldots, n_{i}+1, \ldots, n_{j}-1, \ldots, n_{r}}\right)$ for $2 \leq k \leq n / 2$. Owing to Definition 5, we can deduce that $\operatorname{ME}\left(K_{n_{1}, \ldots, n_{i}, \ldots, n_{j}, \ldots, n_{r}}\right)<$ $\operatorname{ME}\left(K_{n_{1}, \ldots, n_{i}+1, \ldots, n_{j}-1, \ldots, n_{r}}\right)$. It is a contradiction. Hence, for any $i \neq j$, we have $\left|n_{i}-n_{j}\right| \leq 1$. It means that each parameter $n_{i}$ 
equals either $\lfloor n / r\rfloor$ or $\lfloor n / r\rfloor+1$, i.e., $K_{n_{1}, n_{2}, \ldots, n_{r}}$ is isomorphic to $T(n, r)$

\section{Proofs of Theorems 3 and 4}

We first review some useful results of the matching polynomial as the following lemmas.

Lemma 7 (see [5]). Let $u$ be a vertex of $G$. Then

$$
m(G, x)=x m(G-u, x)-\sum_{u \sim v} m(G-u-v, x)
$$

In [33], Gutman showed some parallel results for the roots of the matching polynomial and the spectra of the characteristic polynomial.

Lemma 8 (see [33]). If $H$ is a subgraph of $G$, then $\lambda_{1}(G) \geq$ $\lambda_{1}(H)$. If $G$ is connected and $H$ is a proper subgraph of $G$, then $\lambda_{1}(G)>\lambda_{1}(H)$.

Next, we recall a definition which is introduced in [34].

Definition 9 (see [34]). Define $G_{1}>G_{2}$ if for all $x \geq \lambda_{1}\left(G_{1}\right)$ we have $m\left(G_{2}, x\right)>m\left(G_{1}, x\right)$.

We then list some useful propositions about $>$ which are proved in [34].

Proposition 10 (see [34]). The relation $>$ is transitive. And if $G_{1}>G_{2}$, then $\lambda_{1}\left(G_{1}\right)>\lambda_{1}\left(G_{2}\right)$.

Proposition 11 (see [34]). If $G$ is connected and $H$ is a proper spanning subgraph of $G$, then $G>H$.

Now, we are ready to give the following lemma which is the key to the proofs of Theorems 3 and 4 .

Lemma 12. For a completer-partite graph $K_{n_{1}, \ldots, n_{i}, \ldots, n_{i}, \ldots, n_{r}}$ with $n_{i}-2 \geq n_{j}$, we have $K_{n_{1}, \ldots, n_{i}-1, \ldots, n_{j}+1, \ldots, n_{r}}>K_{n_{1}, \ldots, n_{i}, \ldots, n_{j}, \ldots, n_{r}}$.

Without loss of the generality, we just need to prove the following lemma.

Lemma 13. For a complete $r$-partite graph $K_{n_{1}+1, n_{2}, \ldots, n_{r}}$ with $n_{1}-1 \geq n_{2}$, we have $K_{n_{1}, n_{2}+1, \ldots, n_{r}}>K_{n_{1}+1, n_{2}, \ldots, n_{r}}$.

Proof. Using Lemma 7, we get the following two equations:

$$
\begin{aligned}
m\left(K_{n_{1}+1, n_{2}, \ldots, n_{r}}, x\right)= & x m\left(K_{n_{1}, n_{2}, \ldots, n_{r}}, x\right) \\
& -n_{2} m\left(K_{n_{1}, n_{2}-1, \ldots, n_{r}}, x\right)-\cdots \\
& -n_{r} m\left(K_{n_{1}, n_{2}, \ldots, n_{r}-1}, x\right) . \\
m\left(K_{n_{1}, n_{2}+1, \ldots, n_{r}}, x\right)= & x m\left(K_{n_{1}, n_{2}, \ldots, n_{r}}, x\right) \\
& -n_{1} m\left(K_{n_{1}-1, n_{2}, \ldots, n_{r}}, x\right)-\cdots \\
& -n_{r} m\left(K_{n_{1}, n_{2}, \ldots, n_{r}-1}, x\right) .
\end{aligned}
$$

By subtraction, we deduce that

$$
\begin{aligned}
& m\left(K_{n_{1}+1, n_{2}, \ldots, n_{r}}, x\right)-m\left(K_{n_{1}, n_{2}+1, \ldots, n_{r}}, x\right) \\
& \quad=n_{1} m\left(K_{n_{1}-1, n_{2}, \ldots, n_{r}}, x\right)-n_{2} m\left(K_{n_{1}, n_{2}-1, \ldots, n_{r}}, x\right) .
\end{aligned}
$$

By Definition 9, it suffices to show that, for every $x \geq$ $\lambda_{1}\left(K_{n_{1}, n_{2}+1, \ldots, n_{r}}\right), m\left(K_{n_{1}+1, n_{2}, \ldots, n_{r}}, x\right)-m\left(K_{n_{1}, n_{2}+1, \ldots, n_{r}}, x\right)>0$. In the sequence, we will study the structure of the polynomial (6) and deduce an important recurrent formula.

By Lemma 7, we first give two different expansions of $m\left(K_{n_{1}, n_{2}, \ldots, n_{r}}, x\right)$.

$$
\begin{aligned}
m\left(K_{n_{1}, n_{2}, \ldots, n_{r}}, x\right)= & x m\left(K_{n_{1}-1, n_{2}, \ldots, n_{r}}, x\right) \\
& -n_{2} m\left(K_{n_{1}-1, n_{2}-1, \ldots, n_{r}}, x\right)-\cdots \\
& -n_{r} m\left(K_{n_{1}-1, n_{2}, \ldots, n_{r}-1}, x\right) . \\
m\left(K_{n_{1}, n_{2}, \ldots, n_{r}}, x\right)= & x m\left(K_{n_{1}, n_{2}-1, \ldots, n_{r}}, x\right) \\
& -n_{1} m\left(K_{n_{1}-1, n_{2}-1, \ldots, n_{r}}, x\right)-\cdots \\
& -n_{r} m\left(K_{n_{1}, n_{2}-1, \ldots, n_{r}-1}, x\right) .
\end{aligned}
$$

Then, combining with (7) and (8), we derive that

$$
\begin{aligned}
x & \left(n_{1} m\left(K_{n_{1}-1, n_{2}, \ldots, n_{r}}, x\right)-n_{2} m\left(K_{n_{1}, n_{2}-1, \ldots, n_{r}}, x\right)\right)=\left(n_{1}\right. \\
& \left.-n_{2}\right) m\left(K_{n_{1}, n_{2}, \ldots, n_{r}}, x\right) \\
& +n_{3}\left[n_{1} m\left(K_{n_{1}-1, n_{2}, n_{3}-1, \ldots, n_{r}}, x\right)\right. \\
& \left.-n_{2} m\left(K_{n_{1}, n_{2}-1, n_{3}-1, \ldots, n_{r}}, x\right)\right]+\cdots \\
& +n_{r}\left[n_{1} m\left(K_{n_{1}-1, n_{2}, \ldots, n_{r}-1}, x\right)\right. \\
& \left.-n_{2} m\left(K_{n_{1}, n_{2}-1, \ldots, n_{r}-1}, x\right)\right] .
\end{aligned}
$$

Suppose $x \neq 0$, we get the following recurrent formula of the right side of (6):

$$
\begin{array}{rl}
n_{1} & m\left(K_{n_{1}-1, n_{2}, \ldots, n_{r}}, x\right)-n_{2} m\left(K_{n_{1}, n_{2}-1, \ldots, n_{r}}, x\right) \\
& =\frac{\left(n_{1}-n_{2}\right)}{x} m\left(K_{n_{1}, n_{2}, \ldots, n_{r}}, x\right) \\
& +\frac{n_{3}}{x}\left[n_{1} m\left(K_{n_{1}-1, n_{2}, n_{3}-1, \ldots, n_{r}}, x\right)\right. \\
& \left.-n_{2} m\left(K_{n_{1}, n_{2}-1, n_{3}-1, \ldots, n_{r}}, x\right)\right]+\cdots \\
& +\frac{n_{r}}{x}\left[n_{1} m\left(K_{n_{1}-1, n_{2}, \ldots, n_{r}-1}, x\right)\right. \\
& \left.-n_{2} m\left(K_{n_{1}, n_{2}-1, \ldots, n_{r}-1}, x\right)\right] .
\end{array}
$$

Next, we will repeatedly use the above recurrent formula (10). For convenience, we introduce a new notation 
$\Delta\left(n_{3}, \ldots, n_{r}\right)=n_{1} m\left(K_{n_{1}-1, n_{2}, \ldots, n_{r}}, x\right)-n_{2} m\left(K_{n_{1}, n_{2}-1, \ldots, n_{r}}, x\right)$. Then, (10) can be rewritten as

$$
\begin{aligned}
\Delta\left(n_{3}, \ldots, n_{r}\right)= & \frac{\left(n_{1}-n_{2}\right)}{x} m\left(K_{n_{1}, n_{2}, \ldots, n_{r}}, x\right) \\
& +\frac{n_{3}}{x} \Delta\left(n_{3}-1, \ldots, n_{r}\right)+\cdots \\
& +\frac{n_{r}}{x} \Delta\left(n_{3}, \ldots, n_{r}-1\right) .
\end{aligned}
$$

Let $T=n_{3}+\ldots+n_{r}$. For any $2 \leq h \leq T$, we claim that

$$
\begin{aligned}
& \Delta\left(n_{3}, \ldots, n_{r}\right)=\frac{n_{1}-n_{2}}{x} \sum_{\substack{k=0 \\
i_{3} \in\left\{0,1, \ldots, n_{3}\right\} \\
i_{3} \in i_{r} \in\left\{0,1, \ldots, n_{r}\right\}}} \frac{\left(n_{3}\right)_{i_{3}} \cdots\left(n_{r}\right)_{i_{r}}}{x^{k}} \\
& +\left(\begin{array}{c}
k \\
i_{3}, i_{4}, \ldots, i_{r}
\end{array}\right) m\left(K_{n_{1}, n_{2}, n_{3}-i_{3}, \ldots, n_{r}-i_{r}}, x\right) \\
& +\sum_{\substack{i_{3}+\ldots+i_{r}=h \\
i_{3} \in\left\{0,1, \ldots, n_{3}\right\} \\
i_{r} \in\left\{0,1, \ldots, n_{r}\right\}}} \frac{\left(n_{3}\right)_{i_{3}} \cdots\left(n_{r}\right)_{i_{r}}}{x^{h}} \\
& \quad\left(\begin{array}{c}
h \\
i_{3}, i_{4}, \ldots, i_{r}
\end{array}\right) \Delta\left(n_{3}-i_{3}, \ldots, n_{r}-i_{r}\right) .
\end{aligned}
$$

In the above formula, the symbol $(t)_{s}$, where $s, t$ are two nonnegative integers, is called the falling factorial which is defined as $(t)_{s}=t(t-1)(t-2) \cdots(t-s+1)$, and it means the number of $s$-permutations of $t$ in combinatorics. The symbol $t$ ! is defined as $t !=t(t-1) \cdots 1$. Note that $(t)_{0}=1,0 !=1,(t)_{s}=0$ if $s>t$. The numbers $\left(\underset{i}{i, i_{4}, \ldots, i_{r}}\right)$ in the above formula are the multinomial coefficients and $\left(\begin{array}{c}m \\ i_{3}, i_{4}, \ldots, i_{r}\end{array}\right)=m ! / i_{3} ! i_{4} ! \cdots i_{r} !$. In the reminder of paper, for the brevity of deduction, we give the following notes. If $s$ is a negative integer, then $(t)_{s}=0$. If there exists a number $i_{j}<0$ for $3 \leq j \leq r$, then $\left(\underset{i_{3}, i_{4}, \ldots, i_{r}}{m}\right)=0$.

Now, we prove Equation (12) by induction.

When $h=1$, it is (11). Suppose the above equation is true for $1 \leq h=l<T$. We now consider the case $h=l+1$.

For $\Delta\left(n_{3}-i_{3}, \ldots, n_{r}-i_{r}\right)$, by (11), we can get its expanded formula.

$$
\begin{aligned}
\Delta\left(n_{3}-i_{3}, \ldots, n_{r}-i_{r}\right) & \\
= & \frac{n_{1}-n_{2}}{x} m\left(K_{n_{1}, n_{2}, n_{3}-i_{3}, \ldots, n_{r}-i_{r}}, x\right) \\
& +\frac{n_{3}-i_{3}}{x} \Delta\left(n_{3}-i_{3}-1, \ldots, n_{r}-i_{r}\right)+\cdots \\
& +\frac{n_{r}-i_{r}}{x} \Delta\left(n_{3}-i_{3}, \ldots, n_{r}-i_{r}-1\right) .
\end{aligned}
$$

Then we can obtain that

$$
\begin{aligned}
& \sum_{\substack{i_{3}+\ldots+i_{r}=l \\
i_{3} \in\left\{0,1, \ldots, n_{3}\right\} \\
i_{r} \in\left\{0,1, \ldots, n_{r}\right\}}} \frac{\left(n_{3}\right)_{i_{3}} \cdots\left(n_{r}\right)_{i_{r}}}{x^{l}}\left(\begin{array}{c}
l \\
i_{3}, \ldots, i_{r}
\end{array}\right) \Delta\left(n_{3}\right. \\
& \left.-i_{3}, \ldots, n_{r}-i_{r}\right)=\sum_{\substack{i_{3}+\ldots+i_{r}=l \\
i_{3} \in\left\{0,1, \ldots, n_{3}\right\} \\
i_{r} \in\left\{0,1, \ldots, n_{r}\right\}}} \frac{\left(n_{3}\right)_{i_{3}} \cdots\left(n_{r}\right)_{i_{r}}}{x^{l}}
\end{aligned}
$$

$$
\begin{aligned}
& \cdot\left(\begin{array}{c}
l \\
i_{3}, \ldots, i_{r}
\end{array}\right)\left\{\frac{n_{1}-n_{2}}{x} m\left(K_{n_{1}, n_{2}, n_{3}-i_{3}, \ldots, n_{r}-i_{r}}, x\right)\right. \\
& +\frac{n_{3}-i_{3}}{x} \Delta\left(n_{3}-i_{3}-1, \ldots, n_{r}-i_{r}\right)+\cdots \\
& \left.+\frac{n_{r}-i_{r}}{x} \Delta\left(n_{3}-i_{3}, \ldots, n_{r}-i_{r}-1\right)\right\} .
\end{aligned}
$$

By expanding the last formula, we get that

$$
\begin{aligned}
& \sum_{\substack{i_{3}+\ldots+i_{r}=l \\
i_{3} \in\left\{0,1, \ldots, n_{3}\right\} \\
i_{r} \in\left\{0,1, \ldots, n_{r}\right\}}} \frac{\left(n_{3}\right)_{i_{3}} \cdots\left(n_{r}\right)_{i_{r}}}{x^{l}}\left(\begin{array}{c}
l \\
i_{3}, \ldots, i_{r}
\end{array}\right) \Delta\left(n_{3}\right. \\
& \left.-i_{3}, \ldots, n_{r}-i_{r}\right)=\frac{n_{1}-n_{2}}{x} \\
& \sum_{\substack{i_{3}+\ldots+i_{r}=l \\
i_{3} \in\left\{0,1, \ldots, n_{3}\right\} \\
i_{r} \in\left\{0,1, \ldots, n_{r}\right\}}} \frac{\left(n_{3}\right)_{i_{3}} \cdots\left(n_{r}\right)_{i_{r}}}{x^{l}} \\
& \cdot\left(\begin{array}{c}
l \\
i_{3}, \ldots, i_{r}
\end{array}\right) m\left(K_{n_{1}, n_{2}, n_{3}-i_{3}, \ldots, n_{r}-i_{r}}, x\right) \\
& +\sum_{\substack{i_{3}+\ldots+i_{r}=l \\
i_{3} \in\left\{0,1, \ldots, n_{3}\right\} \\
i_{r} \in\left\{0,1, \ldots, n_{r}\right\}}} \frac{\left(n_{3}\right)_{i_{3}} \cdots\left(n_{r}\right)_{i_{r}}}{x^{l}} \\
& \cdot\left(\begin{array}{c}
l \\
i_{3}, \ldots, i_{r}
\end{array}\right)\left\{\frac{n_{3}-i_{3}}{x} \Delta\left(n_{3}-i_{3}-1, \ldots, n_{r}-i_{r}\right)\right. \\
& \left.+\cdots+\frac{n_{r}-i_{r}}{x} \Delta\left(n_{3}-i_{3}, \ldots, n_{r}-i_{r}-1\right)\right\} .
\end{aligned}
$$

We observe that the general term in the last summation is $\Delta\left(n_{3}-j_{3}, \ldots, n_{r}-j_{r}\right)$, in which $j_{3}, \ldots, j_{r}$ are nonnegative integers and $j_{3}+\ldots+j_{r}=l+1$. We then study the coefficient of $\Delta\left(n_{3}-j_{3}, \ldots, n_{r}-j_{r}\right)$.

By analyzing the above formula, we find that the coefficient of $\Delta\left(n_{3}-j_{3}, \ldots, n_{r}-j_{r}\right)$ consists of $r-2$ parts. For 
every $3 \leq k \leq r$, the $k$ th part comes from the expansion of $\Delta\left(n_{3}-j_{3}, \ldots, n_{k}-j_{k}+1 \ldots, n_{r}-j_{r}\right)$, and it is

$$
\frac{\left(n_{3}\right)_{j_{3}} \cdots\left(n_{r}\right)_{j_{r}}}{x^{l+1}}\left(\begin{array}{c}
l \\
j_{3}, \ldots, j_{k}-1, \ldots, j_{r}
\end{array}\right) .
$$

Thus the coefficient of $\Delta\left(n_{3}-j_{3}, \ldots, n_{r}-j_{r}\right)$ is

$$
\begin{aligned}
& \frac{\left(n_{3}\right)_{j_{3}} \cdots\left(n_{r}\right)_{j_{r}}}{x^{l+1}}\left\{\left(\begin{array}{c}
l \\
j_{3}-1, j_{4}, \ldots, j_{r}
\end{array}\right)\right. \\
& \left.\quad+\left(\begin{array}{c}
l \\
j_{3}, j_{4}-1, \ldots, j_{r}
\end{array}\right)+\ldots+\left(\begin{array}{c}
l \\
j_{3}, j_{4}, \ldots, j_{r}-1
\end{array}\right)\right\} .
\end{aligned}
$$

It is well known that

$$
\begin{array}{r}
\left(\begin{array}{c}
l \\
j_{3}-1, j_{4}, \ldots, j_{r}
\end{array}\right)+\left(\begin{array}{c}
l \\
j_{3}, j_{4}-1, \ldots, j_{r}
\end{array}\right)+\ldots \\
+\left(\begin{array}{c}
l \\
j_{3}, j_{4}, \ldots, j_{r}-1
\end{array}\right)=\left(\begin{array}{c}
l+1 \\
j_{3}, j_{4}, \ldots, j_{r}
\end{array}\right) .
\end{array}
$$

It follows the coefficient of $\Delta\left(n_{3}-j_{3}, \ldots, n_{r}-j_{r}\right)$ is equal to

$$
\frac{\left(n_{3}\right)_{j_{3}} \cdots\left(n_{r}\right)_{j_{r}}}{x^{l+1}}\left(\begin{array}{c}
l+1 \\
j_{3}, j_{4}, \ldots, j_{r}
\end{array}\right) \text {. }
$$

Owing to the above analysis, we conclude that

$$
\begin{aligned}
& \sum_{\substack{i_{3}+\ldots+i_{r}=l \\
i_{3} \in\left\{0,1, \ldots, n_{3}\right\} \\
i_{r} \in\left\{0,1, \ldots, n_{r}\right\}}} \frac{\left(n_{3}\right)_{i_{3}} \cdots\left(n_{r}\right)_{i_{r}}}{x^{l}} \\
& \cdot\left(\begin{array}{c}
l \\
i_{3}, \ldots, i_{r}
\end{array}\right) \Delta\left(n_{3}-i_{3}, \ldots, n_{r}-i_{r}\right)=\frac{n_{1}-n_{2}}{x} \\
& \cdot \sum_{\substack{i_{3}+\ldots+i_{r}=l \\
i_{3} \in\left\{0,1, \ldots, n_{3}\right\} \\
i_{r} \in\left\{0,1, \ldots, n_{r}\right\}}} \frac{\left(n_{3}\right)_{i_{3}} \cdots\left(n_{r}\right)_{i_{r}}}{x^{l}} \\
& \cdot\left(\begin{array}{c}
l \\
i_{3}, \ldots, i_{r}
\end{array}\right) m\left(K_{\left.n_{1}, n_{2}, n_{3}-i_{3}, \ldots, n_{r}-i_{r}, x\right)}\right. \\
& +\sum_{\substack{i_{3}+\ldots+i_{r}=l+1 \\
i_{3} \in\left\{0,1, \ldots, n_{3}\right\} \\
i_{r} \in\left\{0,1, \ldots, n_{r}\right\}}} \frac{\left(n_{3}\right)_{i_{3}} \cdots\left(n_{r}\right)_{i_{r}}}{x^{l+1}} \\
& \cdot\left(\begin{array}{c}
l+1 \\
i_{3}, \ldots, i_{r}
\end{array}\right) \Delta\left(n_{3}-i_{3}, \ldots, n_{r}-i_{r}\right) .
\end{aligned}
$$

Hence, (12) holds for $h=l+1$. By induction, (12) is true for any $2 \leq h \leq T$.
When $h=T$, we get the final expanded form.

$$
\begin{aligned}
& m\left(K_{n_{1}+1, n_{2}, \ldots, n_{r},}, x\right)-m\left(K_{\left.n_{1}, n_{2}+1, \ldots, n_{r}, x\right)}\right.
\end{aligned}
$$

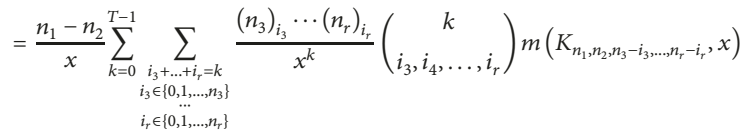

$$
\begin{aligned}
& +\frac{n_{3} ! \cdots n_{r} !}{x^{T}}\left(\begin{array}{c}
T \\
n_{3}, n_{4}, \ldots, n_{r}
\end{array}\right) \Delta(0, \ldots, 0)
\end{aligned}
$$

Note that $\Delta(0, \ldots, 0)=n_{1} m\left(K_{n_{1}-1, n_{2}}, x\right)-n_{2} m\left(K_{n_{1}, n_{2}-1}, x\right)$. Now, considering the element $n_{1} m\left(K_{n_{1}-1, n_{2}}, x\right)$ $n_{2} m\left(K_{n_{1}, n_{2}-1}, x\right)$, for $x \neq 0$, we have

$$
\begin{aligned}
n_{1} m & \left(K_{n_{1}-1, n_{2}}, x\right)-n_{2} m\left(K_{n_{1}, n_{2}-1}, x\right) \\
= & n_{1} \sum_{k \geq 0} x^{n_{1}+n_{2}-1-2 k}(-1)^{k} m\left(K_{n_{1}-1, n_{2}}, k\right) \\
& -n_{2} \sum_{k \geq 0} x^{n_{1}+n_{2}-1-2 k}(-1)^{k} m\left(K_{n_{1}, n_{2}-1}, k\right) \\
& =\sum_{k \geq 0} x^{n_{1}+n_{2}-1-2 k}(-1)^{k} \\
& \cdot\left[\frac{n_{1}\left(n_{1}-1\right)_{k}\left(n_{2}\right)_{k}}{k !}-\frac{n_{2}\left(n_{1}\right)_{k}\left(n_{2}-1\right)_{k}}{k !}\right] \\
= & \sum_{k \geq 0} x^{n_{1}+n_{2}-1-2 k}(-1)^{k}\left(n_{1}-n_{2}\right) \frac{\left(n_{1}\right)_{k}\left(n_{2}\right)_{k}}{k !} \\
= & \sum_{k \geq 0} x^{n_{1}+n_{2}-1-2 k}(-1)^{k}\left(n_{1}-n_{2}\right) m\left(K_{n_{1}, n_{2}}, k\right) \\
= & \frac{n_{1}-n_{2}}{x} m\left(K_{n_{1}, n_{2}}, x\right) .
\end{aligned}
$$

From the above calculations, for $x \neq 0$, we can deduce the following result:

$$
\begin{gathered}
m\left(K_{n_{1}+1, n_{2}, \ldots, n_{r}}, x\right)-m\left(K_{n_{1}, n_{2}+1, \ldots, n_{r}}, x\right)=\frac{n_{1}-n_{2}}{x} \\
\cdot \sum_{\substack{k=0 \\
i_{3}+\ldots+i_{i}=k \\
i_{3} \in\left\{0,1, \ldots, n_{3}\right\} \\
i_{r} \in\left\{0, \ldots, \ldots, n_{r}\right\}}} \frac{\left(n_{3}\right)_{i_{3}} \cdots\left(n_{r}\right)_{i_{r}}}{x^{k}}
\end{gathered}
$$$$
\cdot\left(\begin{array}{c}
k \\
i_{3}, i_{4}, \ldots, i_{r}
\end{array}\right) m\left(K_{n_{1}, n_{2}, n_{3}-i_{3}, \ldots, n_{r}-i_{r}}, x\right) .
$$

Thus,

$$
\begin{gathered}
x^{T+1}\left[m\left(K_{n_{1}+1, n_{2}, \ldots, n_{r}}, x\right)-m\left(K_{n_{1}, n_{2}+1, \ldots, n_{r}}, x\right)\right]=\left(n_{1}\right. \\
\left.-n_{2}\right) \sum_{\substack{k=0 \\
i_{3} \in\left\{0,1, \ldots, n_{3}\right\} \\
i_{r} \in\left\{0,1, \ldots, n_{r}\right\}}}^{T}\left(n_{3}\right)_{i_{3}} \cdots\left(n_{r}\right)_{i_{r}} \\
\cdot x^{T-k}\left(\begin{array}{c}
k \\
i_{3}, i_{4}, \ldots, i_{r}
\end{array}\right) m\left(K_{n_{1}, n_{2}, n_{3}-i_{3}, \ldots, n_{r}-i_{r}}, x\right) .
\end{gathered}
$$


For a set of nonnegative integers $\left\{i_{3}, \ldots i_{r}\right\}$, $K_{n_{1}, n_{2}, n_{3}-i_{3}, \ldots, n_{r}-i_{r}}$ is a proper subgraph of $K_{n_{1}, n_{2}+1, \ldots, n_{r}}$. By Lemma 8, we get $\lambda_{1}\left(K_{n_{1}, n_{2}+1, \ldots, n_{r}}\right)>\lambda_{1}\left(K_{n_{1}, n_{2}, n_{3}-i_{3}, \ldots, n_{r}-i_{r}}\right)$. When $x \geq \lambda_{1}\left(K_{n_{1}, n_{2}+1, \ldots, n_{r}}\right)$, we have $m\left(K_{n_{1}, n_{2}, n_{3}-i_{3}, \ldots, n_{r}-i_{r}}, x\right)>$ 0 . By (24), we have $m\left(K_{n_{1}+1, n_{2}, \ldots, n_{r}}, x\right)-m\left(K_{n_{1}, n_{2}+1, \ldots, n_{r}}, x\right)>0$. It follows that $K_{n_{1}, n_{2}+1, \ldots, n_{r}}>K_{n_{1}+1, n_{2}, \ldots, n_{r}}$.

We thus complete the proof.

Now, it is time to prove Theorems 3 and 4 .

Proof of Theorem 3. Suppose that the largest matching root of complete $r$-partite graphs is minimal for $K_{n_{1}, n_{2}, \ldots, n_{r}}$. If there are two parameters $n_{i}$ and $n_{j}$ satisfying $n_{j} \geq n_{i}>$ 1 , then $\left(n_{j}+1\right)-\left(n_{i}-1\right) \geq 2$. From Lemma 12, we get $K_{n_{1}, \ldots, n_{i}, \ldots, n_{j}, \ldots, n_{r}}>K_{n_{1}, \ldots, n_{i}-1, \ldots, n_{j}+1, \ldots, n_{r}}$. By Proposition 10, we get $\lambda_{1}\left(K_{n_{1}, \ldots, n_{i}, \ldots, n_{j}, \ldots, n_{r}}\right)>\lambda_{1}\left(K_{n_{1}, \ldots, n_{i}-1, \ldots, n_{j}+1, \ldots, n_{r}}\right)$. It is a contradiction. Hence, $K_{n_{1}, n_{2}, \ldots, n_{r}}$ satisfies that all parameters are equal to 1 except one parameter equal to $n-r+1$. Thus, $K_{n_{1}, n_{2}, \ldots, n_{r}} \cong C S(n, r-1)$.

Proof of Theorem 4. Suppose that the largest matching root of complete $r$-partite graphs is maximal for $K_{n_{1}, n_{2}, \ldots, n_{r}}$. If there are two parameters $n_{i}$ and $n_{j}$ satisfying $n_{j}, \geq n_{i}+$ 2 , then $n_{j}-1 \geq 1$. Owing to Lemma 12, we have $K_{n_{1}, \ldots, n_{i}+1, \ldots, n_{j}-1, \ldots, n_{r}}>K_{n_{1}, \ldots, n_{i}, \ldots, n_{j}, \ldots, n_{r}}$. By Proposition 10, it implies that $\lambda_{1}\left(K_{n_{1}, \ldots, n_{i}+1, \ldots, n_{j}-1, \ldots, n_{r}}\right)>\lambda_{1}\left(K_{n_{1}, \ldots, n_{i}, \ldots, n_{j}, \ldots, n_{r}}\right)$. It is a contradiction. Hence, for any $i \neq j$, we have $\left|n_{i}-n_{j}\right| \leq 1$. It means that each parameter $n_{i}$ equals either $\lfloor n / r\rfloor$ or $\lfloor n / r\rfloor+1$, i.e., $K_{n_{1}, n_{2}, \ldots, n_{r}}$ is isomorphic to $T(n, r)$.

\section{Conclusion}

Recently, there are many results about the matching energy and matching roots of graphs $[7,11,12,18-23,25-28,31,34]$, due to their important applications in statistical physics and chemistry. In this paper, we investigate the matching energy and the largest matching root among all the complete $r$ partite graphs with given order $n$, respectively. We find that their minimal graphs are both complete split graph $C S(n, r-$ 1 ), and the maximal graphs are both Turán graph $T(n, r)$. In fact, complete split graph and Turán graph are two important graph classes which have extensive applications in extremal graph theory and network. For more details, we refer to [35]. In addition, there are many other chemical invariants, such as Laplacian energy [36], Randić energy [37], and incidence energy [38]. In the future, we would like to characterize the extremal graphs of the above invariants among the complete $r$-partite graphs.

\section{Data Availability}

No data were used to support this study.

\section{Conflicts of Interest}

The authors declare that there are no conflicts of interest regarding the publication of this paper.

\section{Acknowledgments}

This work was supported by National Natural Science Foundation of China (no. 11601511), the Natural Science Foundation of Jiangsu Province (nos. BK20160237 and BK20150169).

\section{References}

[1] E. J. Farrell, “An introduction to matching polynomials," Journal of Combinatorial Theory, Series B, vol. 27, no. 1, pp. 75-86, 1979.

[2] C. D. Godsil and I. Gutman, "On the theory of the matching polynomial," Journal of Graph Theory, vol. 5, no. 2, pp. 137-144, 1981.

[3] I. Gutman, "The matching polynomial," MATCH Communications in Mathematical and in Computer Chemistry, vol. 6, pp. 75-91, 1979.

[4] O. J. Heilmann and E. H. Lieb, "Theory of monomer-dimer systems," Communications in Mathematical Physics, vol. 25, pp. 190-232, 1972.

[5] C. Godsil, Algebraic Combinatorics, Chapman and Hall, London, UK, 1993.

[6] D. C. Fisher and J. Ryan, "Bounds on the largest root of the matching polynomial," Discrete Mathematics, vol. 110, no. 1-3, pp. 275-278, 1992.

[7] S. Akbari, P. Csikvári, A. Ghafari, S. Khalashi Ghezelahmad, and M. Nahvi, "Graphs with integer matching polynomial zeros," Discrete Applied Mathematics: The Journal of Combinatorial Algorithms, Informatics and Computational Sciences, vol. 224, pp. 1-8, 2017.

[8] E. Ghorbani, "Graphs with few matching roots," Graphs and Combinatorics, vol. 29, no. 5, pp. 1377-1389, 2013.

[9] I. Gutman, "A survey on the matching polynomial," in Graph Polynomials, M. Dehmer, Y. Shi, I. Gutman, and X. Li, Eds., pp. 73-95, CRC Press, Boca Raton, FL, USA, 2016.

[10] W. Liu, Q. Guo, Y. Zhang, L. Feng, and I. Gutman, "Further results on the largest matching root of unicyclic graphs," Discrete Applied Mathematics: The Journal of Combinatorial Algorithms, Informatics and Computational Sciences, vol. 221, pp. 82-88, 2017.

[11] H. Zhang, R. Lin, and G. Yu, "The largest matching root of unicyclic graphs," Information Processing Letters, vol. 113, no. 1921, pp. 804-806, 2013.

[12] H. Zhang, G. Yu, and S. Li, "Graphs with six distinct matching roots," Information Processing Letters, vol. 115, no. 5, pp. 521-526, 2015.

[13] I. Gutman and S. Wagner, "The matching energy of a graph," Discrete Applied Mathematics, vol. 160, no. 15, pp. 2177-2187, 2012.

[14] I. Gutman, "The energy of a graph," Berichte der MathematischStatistischen Sektion im Forschungszentrum Graz, vol. 103, pp. 1-22, 1978.

[15] X. Li, Y. Shi, and I. Gutman, Graph Energy, Springer, New York, NY, USA, 2012.

[16] I. Gutman, "Acyclic systems with extremal Hückel $\pi$-electron energy," Theoretical Chemistry Accounts, vol. 45, no. 2, pp. 7987, 1977.

[17] I. Gutman, M. Milun, and N. Trinajstić, "Graph theory and molecular orbitals. 19. Nonparametric resonance energies of arbitrary conjugated systems," Journal of the American Chemical Society, vol. 99, no. 6, pp. 1692-1704, 1977. 
[18] L. Chen and J. Liu, "The bipartite unicyclic graphs with the first $\lfloor(n-3) / 4\rfloor$ largest matching energies," Applied Mathematics and Computation, vol. 268, pp. 644-656, 2015.

[19] L. Chen and J. Liu, "Extremal values of matching energies of one class of graphs," Applied Mathematics and Computation, vol. 273, pp. 976-992, 2016.

[20] L. Chen, J. Liu, and Y. Shi, "Matching energy of unicyclic and bicyclic graphs with a given diameter," Complexity, vol. 21, no. 2, pp. 224-238, 2015.

[21] L. Chen, J. Liu, and Y. Shi, "Bounds on the matching energy of unicyclic odd-cycle graphs," MATCH - Communications in Mathematical and in Computer Chemistry, vol. 75, no. 2, pp. 315330, 2016.

[22] L. Chen and Y. Shi, "The maximal matching energy of tricyclic graphs," MATCH Communications in Mathematical and in Computer Chemistry, vol. 73, no. 1, pp. 105-119, 2015.

[23] X. Chen, X. Li, and H. Lian, "The matching energy of random graphs," Discrete Applied Mathematics, vol. 193, pp. 102-109, 2015.

[24] I. Gutman, "Matching energy," in Graph Energies-Theory and Applications, I. Gutman and X. Li, Eds., pp. 167-190, University of Kragujevac, Kragujevac, Serbia, 2016.

[25] S. Ji, X. Li, and Y. Shi, "Extremal matching energy of bicyclic graphs," MATCH Communications in Mathematical and in Computer Chemistry, vol. 70, no. 2, pp. 697-706, 2013.

[26] S. Ji and H. Ma, "The extremal matching energy of graphs," Ars Combinatoria, vol. 115, pp. 343-355, 2014.

[27] H.-H. Li, Y.-X. Zhou, and L. Su, "Graphs with extremal matching energies and prescribed parameters," MATCH - Communications in Mathematical and in Computer Chemistry, vol. 72, no. 1, pp. 239-248, 2014.

[28] S. Li and W. Yan, "The matching energy of graphs with given parameters," Discrete Applied Mathematics, vol. 162, pp. 415420, 2014

[29] J. Monsalve, J. Rada, and Y. Shi, "Extremal values of energy over oriented bicyclic graphs," Applied Mathematics and Computation, vol. 342, pp. 26-34, 2019.

[30] K. Xu, K. C. Das, and Z. Zheng, "The minimal matching energy of $(n, m)$-graphs with a given matching number," MATCH Communications in Mathematical and in Computer Chemistry, vol. 73, no. 1, pp. 93-104, 2015.

[31] K. Xu, Z. Zheng, and K. Das, "Extremal t-apex trees with respect to matching energy," Complexity, vol. 21, no. 5, pp. 238-247, 2016.

[32] D. Stevanovic', I. Gutman, and M. U. Rehman, "On spectral radius and energy of complete multipartite graphs," Ars Mathematica Contemporanea, vol. 9, no. 1, pp. 109-113, 2015.

[33] I. Gutman, "A note on analogies between the characteristic and the matching polynomial of a graph," Publications de l'institut Mathématique, vol. 31, no. 45, pp. 27-31, 1982.

[34] X. Chen, X. Li, and H. Lian, "Solution to a conjecture on the maximum skew-spectral radius of odd-cycle graphs," The Electronic Journal of Combinatorics, vol. 22, no. 1, pp. P1-71, 2015.

[35] A. Branstadt, V. B. Le, and J. P. Spinrad, Graph Classes: A Survey, SIAM Monographs on Discrete Mathematics and Applications, Society for Industrial and Applied Mathematics (SIAM), Philadelphia, PA, USA, 1999.

[36] I. Gutman and B. Zhou, "Laplacian energy of a graph," Linear Algebra and its Applications, vol. 414, no. 1, pp. 29-37, 2006.

[37] I. Gutman, B. Furtula, and S. Bozkurt, "On Randic' energy," Linear Algebra and its Applications, vol. 442, no. 1, pp. 50-57, 2014.
[38] S. B. Bozkurt and D. Bozkurt, "On incidence energy," MATCH Communications in Mathematical and in Computer Chemistry, vol. 72, no. 1, pp. 215-225, 2014. 


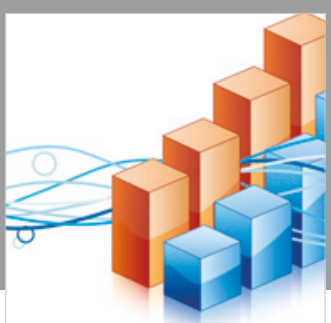

Advances in

Operations Research

\section{-n-m}
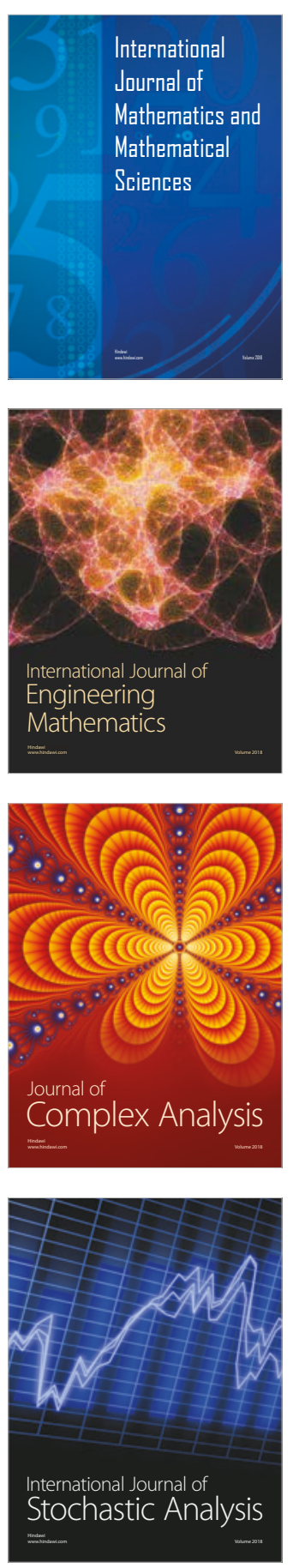
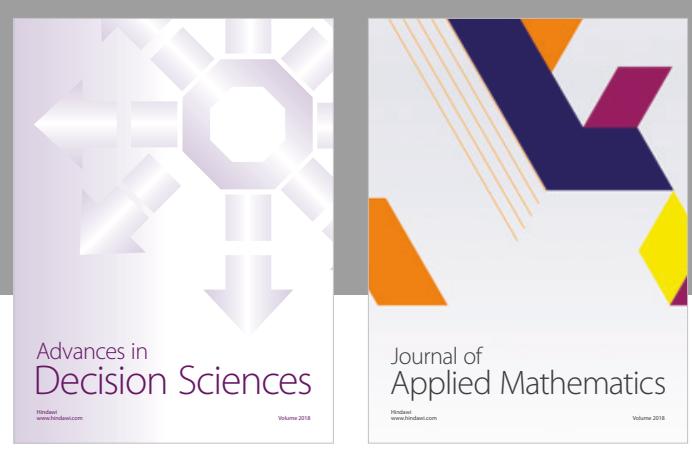

Journal of

Applied Mathematics
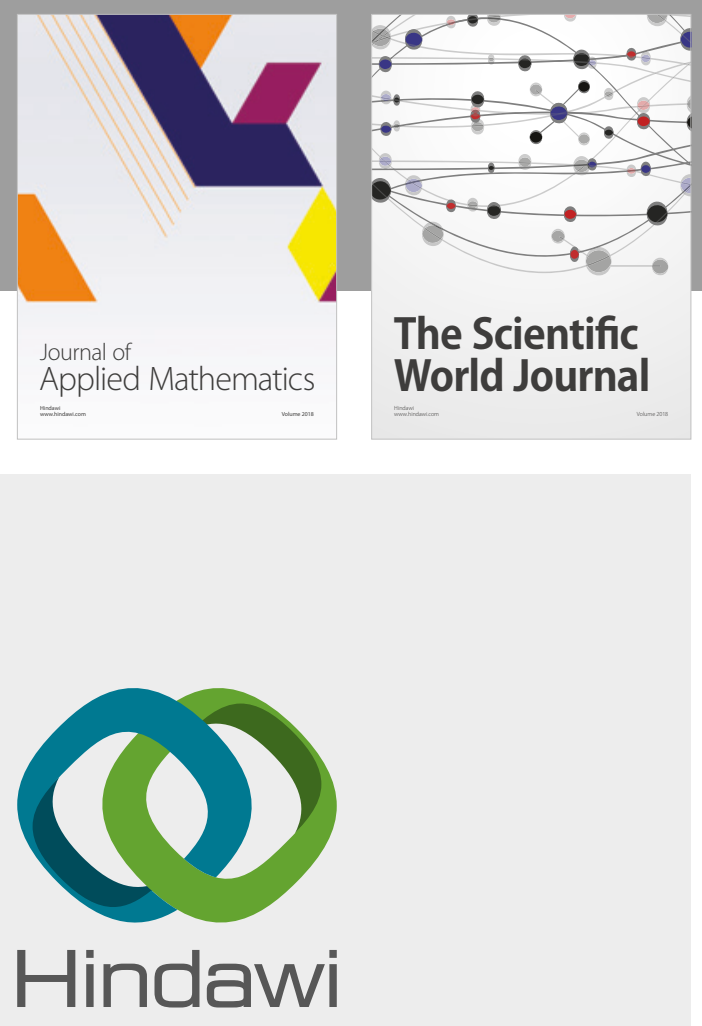

Submit your manuscripts at

www.hindawi.com

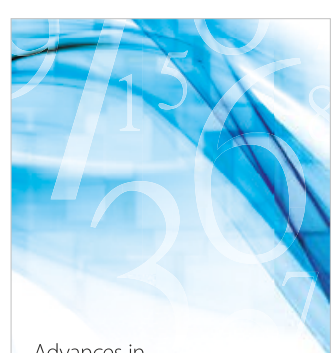

Advances in
Numerical Analysis
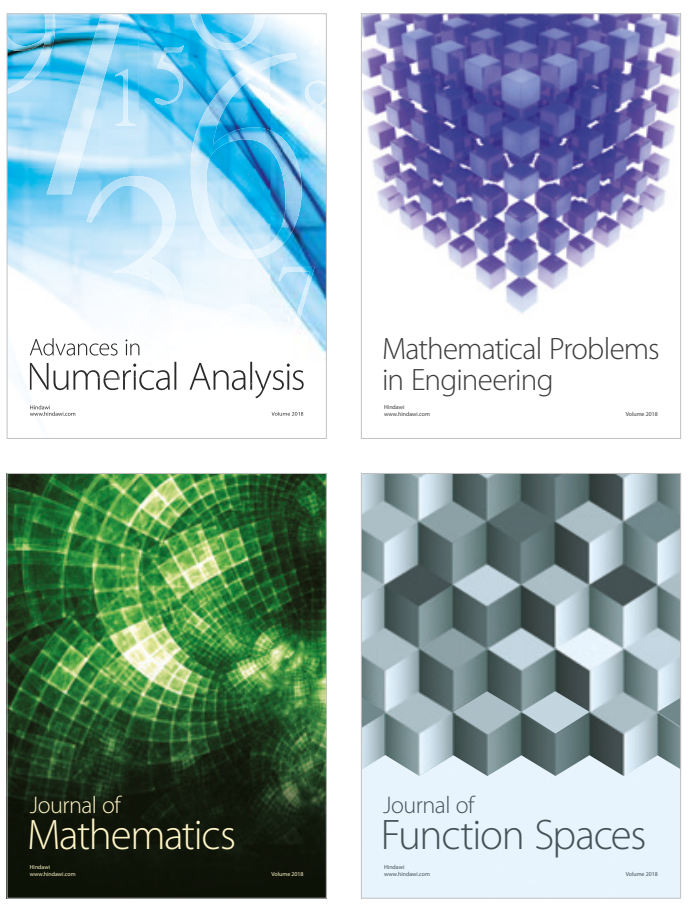

Mathematical Problems in Engineering

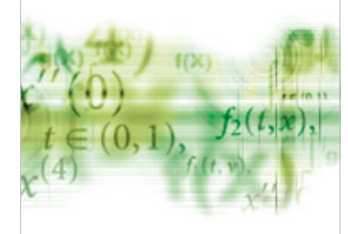

International Journal of

Differential Equations

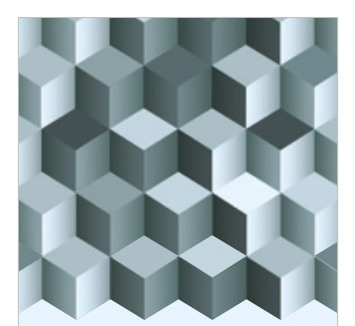

Journal of

Function Spaces
The Scientific

World Journal

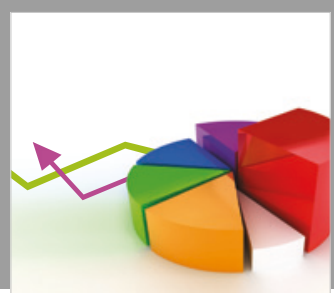

Journal of

Probability and Statistics
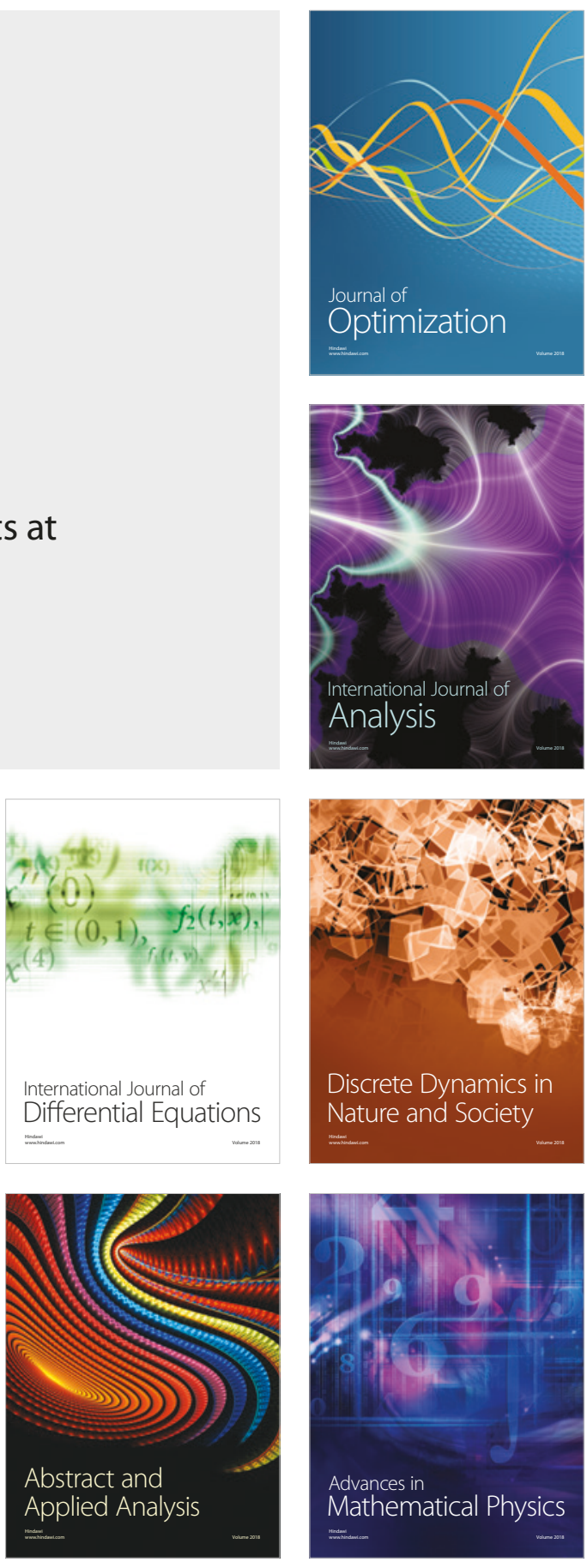